\title{
Clinical Study of Sulfotanshinone Sodium Injection in Treating Non-Ischemic Retinal Vein Occlusion
}

\author{
Bingwen Lu, Xingwei Wu* \\ Ophthalmology Department, Shanghai First People's Hospital, Shanghai, China \\ Email: ${ }^{*} 704487389 @$ qq.com
}

Received 19 March 2015; accepted 8 May 2015; published 12 May 2015

Copyright (C) 2015 by authors and Scientific Research Publishing Inc.

This work is licensed under the Creative Commons Attribution International License (CC BY). http://creativecommons.org/licenses/by/4.0/

(c) (i) Open Access

\begin{abstract}
Objectives: To study the effect of sulfotanshinone sodium (SS) injection in the treatment of nonischemic retinal vein occlusion (RVO). Methods: Sixty-two RVO patients treated in our hospital between Jan. 2013 and Oct. 2014 were randomly divided into Control Group (30 patients; Bendazol tablets) and Treatment Group (32 patients, Bendazol tablets + SS injections), each with a follow-up period of 6 months. Statistical analysis was then performed on changes in visual acuity, central retinal thickness (CRT) and retinal circulation time (RCT) before and after the treatment. Results: After treatment, both Control Group and Treatment Group witnessed an improvement on visual acuity (Control Group: $t=2.103, p=0.044$; Treatment Group: $t=8.021, p=0.000$ ). Visual acuity could be greatly improved in Treatment Group when compared with Control Group, with significant differences $(p<0.01)$. Macular edema could be greatly relieved in Treatment Group measured by CRT ( $t=2.571, p=0.007$ ) while the difference was of no statistical significance in Control Group $(t=1.016, p=0.070)$. $R C$ T were remarkably shortened in both groups (Control Group: $t=$ 43.83, $p=0.000$; Treatment Group: $t=27.34, p=0.000$ ), and when compared with Control group, the changes in Treatment Group were more significant $(p<0.05)$. Conclusion: SS injection could effectively improve the therapeutic effect in patients with non-ischemic retinal vein occlusion.
\end{abstract}

\section{Keywords}

Sulfotanshinone Sodium Injection, Non-Ischemic Retinal Vein Occlusion

\section{Introduction}

Retinal vein occlusion (RVO) is the second most common retinal vascular disorder after diabetic retinopathy "Corresponding author. 
with significant morbidity, including branch retinal vein occlusion (BRVO) and central retinal vein occlusion (CRVO) as well as their ischemic and non-ischemic subtypes [1]. Major causes of vision loss include macular edema and neovascularization with secondary vitreous hemorrhage and/or neovascular glaucoma [2]. Until recently, effective treatment options for RVO were limited, including grid laser photocoagulation, intravitreal injections of triamcindion acetonide and intravitreal injections of anti-vascular endothelial growth factor (VEGF) agents [3].

Tanshinone IIA (TSA) is an herbal monomer with a clear chemical structure, isolated from Danshen (Salvia miltiorrhiza). In Traditional Chinese Medicine (TCM), Danshen is considered to promote blood circulation for removing blood stasis and improve microcirculation, which thus has been widely used to treat cardiovascular diseases for more than 2000 years in China [4]. Over the last decade, interest in the mechanism of its versatile protective effects on neurodegenerative diseases, metabolic abnormalities, and ischemic damages has been growing [5]-[7]. Up till now, few studies have reported on its clinical use in the area of retinal ischemic diseases. This prospective clinical study was designed to assess the safety and efficacy of TSA in treating RVO patients.

\section{Materials and Methods}

\subsection{Research Objects and Grouping}

This prospective, open-label, comparative case series study was conducted at Ophthalmology department of Shanghai First People's Hospital from Jan. 2013 to Oct. 2014. Consecutive cases of RVO patients who could come for regular follow-up visits were invited to participate in the study.

The study group comprised patients who met the eligibility criteria as follows: 1) age of at least 18 years with RVO; 2) best-corrected visual acuity (BCVA) of 30 letters to 90 letters using the ETDRS charts; 3 ) color fundus photography documented thin retinal artery, dilated vein, posterior pole retinal edema and hemorrhage; 4) fundus fluorescence angiography (FFA) featured delayed arterial filling, delayed retinal arterial branches filling, delayed laminar flow in large retinal veins, and no filling or only retrograde filling in retinal vein branches. No capillary non-perfusion (NP) was present in non-ischemic RVO.

Patients were excluded from the study if they had undergone any other treatment for RVO within 3 months, such as laser therapy or intravitreal injections. Patients with age-related macular degeneration, diabetic retinopathy, uncontrolled glaucoma, or ocular inflammation that could compromise visual acuity were excluded. Patients with uncontrolled hypertension, diabetic mellitus, myocardial infarction, or cerebrovascular accident within three months of presentation were also excluded.

Informed consent was obtained from the patients before enrollment in the study. Ethical approval was obtained from the Shanghai First People's Hospital Research Center.

\subsection{Experiment Medicine}

Sixty-two RVO patients were randomized into two groups: Control Group receiving Bdazol tablets only (10 mg each time, three times daily), Treatment Group receiving Bendazol tablets (10 mg each time, three times daily) + sulfotanshinone sodium (SS) injections (20 mg per day, one week consecutively in one month).

SS injection $(5 \mathrm{mg} / \mathrm{ml})$ manufactured by the First Biochemical Pharmaceutical Co. Ltd., Shanghai, China, is now the clinically available TSA agent, approved by State Food and Drug Administration of China. The dosage for administration of SS injection is $20 \mathrm{mg}$ per day. SS injection is given diluted at the point of treatment in 250 $\mathrm{mL} 5 \%$ glucose injection for intravenous administration.

In addition to the treatment medicines, usages of any other traditional Chinese medicine or modern western medicine that affects blood circulation during the 6 months follow-up were prohibited.

\subsection{Experiment Methods}

A detailed history was taken to ascertain each patient's demographics and chief complaints, including duration of the symptom and presence of systemic diseases, such as hypertension, diabetes mellitus, cardiac diseases and hyperlipidemia.

Each patient underwent BCVA measurement with the ETDRS chart. Clinical examination included slit-lamp examination, intraocular pressure measurement using noncontact tonometer (NCT) (Nikon, Japan). Baseline central retinal thickness (CRT) was measured with Optical Coherence Tomography (OCT) (Zeiss, Germany). A 
fluorescein angiogram (FFA) (Heidelberg, Germany) was performed to identify the presence of non-ischemic $\mathrm{RVO}$ and record the retinal circulation time (RCT).

All patients underwent monthly BCVA testing, intraocular pressure measurement and OCT scans. FFA was carried out again at the 6 month.

A flow diagram of the entire trial was presented (Figure 1).

\subsection{Statistical Analysis}

Test results were represented by $\mathrm{x} \pm \mathrm{s}$ using the SPSS 18.0 software. Statistical methods such as t-test for independent samples, paired sample t-test and analysis of variance were used for testing and analysis. P values less than 0.05 were considered statistically significant in this study.

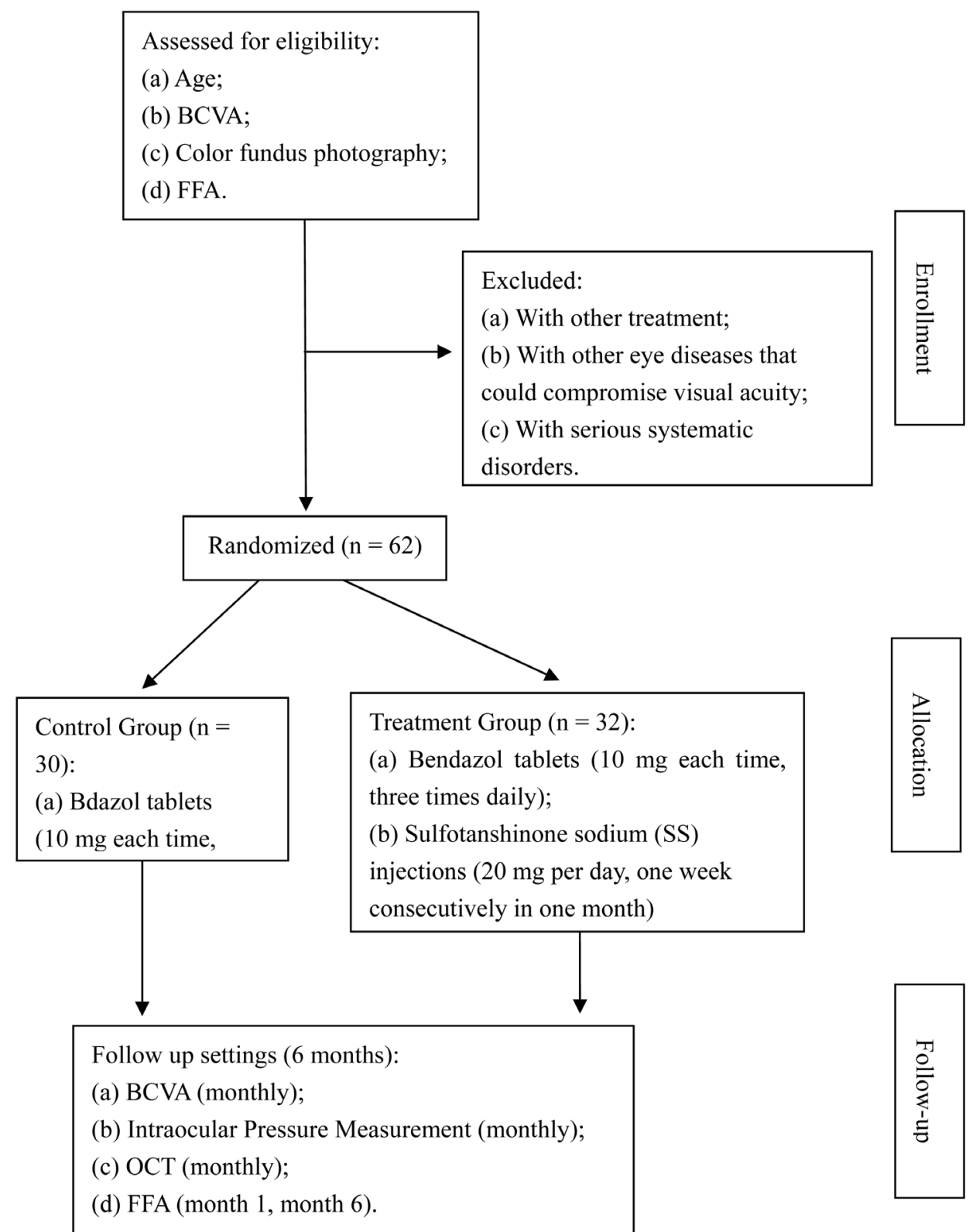

Figure 1. Flow diagram of the progress through the phases of a randomized trial (enrollment, intervention allocation, and follow-up). BCVA: Best-Corrected Visual Acuity; FFA: Fluorescein Angiogram; OCT: Optical Coherence Tomography. 


\section{Results}

\subsection{Patient Demographics and Baseline Characteristics}

A total of 62 eyes of 62 consecutive patients ( 20 men and 42 women) were included in the study. The mean age of the study population was $62 \pm 7$ years (range $50-80$ ). The mean duration of symptoms prior to presentation was $2.8 \pm 1.8$ months with a range of 0.2 months to 6 months. Eighteen eyes had CRVO, and 44 eyes had BRVO. Concurrent systemic hypertension was found in 30 cases $(48.3 \%)$, and nine patients (14.5\%) had diabetic mellitus. Sixty-two patients were randomized into Control Group and Treatment Group. Table 1 summarizes the clinical characteristics of the two groups. Demographic date revealed no statistical difference.

\subsection{Visual Acuity}

RVO patients in Treatment Group had a mean BCVA of $64 \pm 14$ letters before treatment. After the initial 3 months, mean BCVA improved signigicantly, and this gain was maintained at 6 months. Final BCVA was $74 \pm$ 10 letters, which was significantly better than the acuity at baseline $(t=8.021, p=0.000)$. RVO patients in Control Group had a mean BCVA of $65 \pm 13$ before treatment and a final BCVA of $67 \pm 13$ letters. Visual acuity did not significantly improve between baseline and 6 months $(\mathrm{t}=2.103, \mathrm{p}=0.044)$. The improvement of visual acuity was better in Treatment Group when compared with Control group $(p<0.01)$. Table 2 summarizes the changes of BCVA of the two groups ( $\mathrm{x} \pm \mathrm{s}$, letters).

\subsection{Central Retinal Thickness (CRT)}

The mean CRT of RVO patients in Treatment Group at baseline was $406.3 \pm 23.3 \mu \mathrm{m}$ and $338.4 \pm 20.7 \mu \mathrm{m}$ at 6 months. The changes in CRT from baseline were statistically significant $(t=2.571, p=0.007)$. The mean CRT of RVO patients in Control Group at baseline was $385.3 \pm 8.3 \mu \mathrm{m}$ and $342.7 \pm 19.2 \mu \mathrm{m}$ at 6 months. The changes in CRT from baseline were of no statistical importance $(t=1.016, p=0.07)$. The changes of CRT of the two groups were summarized in Table 3 .

\subsection{Retinal Circulation Time (RCT)}

Prolonged RCT was found in both CRVO and BRVO patients. After treatment, the RCT in RVO patients in Treatment Group were reduced to $3.04 \pm 0.14 \mathrm{sec}$ from $5.11 \pm 0.29 \mathrm{sec}$. The changes in RCT from baseline were statistically significant $(t=43.83, p=0.000)$. The mean RCT of RVO patients in Control Group at baseline was $5.15 \pm 0.21 \mathrm{sec}$ and $3.81 \pm 0.13$ at 6 months. Although the changes were also of statistical importance $(\mathrm{t}=27.34$, $\mathrm{p}=0.000)$. When compared with Control Group, the changes in Treatment Group were more significant $(\mathrm{p}<$ 0.05). The changes of RCT of the two groups were summarized in Table 4.

\subsection{Safety}

There were no major ocular or systemic problems, such as increased intraocular pressure, retinal detachment, intraocular inflammation, or vascular events during the 6 months follow-up in both groups.

Table 1. Demographics and clinical characteristics of the two groups.

\begin{tabular}{ccc}
\hline & Control Group & Treatment Group \\
\hline Number of patients & 30 & 32 \\
Age, years & $62 \pm 7$ & $62 \pm 7$ \\
Sex (male/female) & $10 / 20$ & $10 / 22$ \\
Duration of symptoms prior to presentation, months & $2.5 \pm 1.9$ & $3.0 \pm 1.4$ \\
BCVA, letters & $65 \pm 13$ & $64 \pm 14$ \\
CRT, $\mu \mathrm{m}$ & $385.3 \pm 8.3$ & $406.3 \pm 23.3$ \\
RCT, sec & $5.15 \pm 0.21$ & $5.11 \pm 0.29$ \\
\hline
\end{tabular}

Data are mean \pm SD; BCVA: Best-Corrected Visual Acuity; CRT: Central Retinal Thickness; RCT: Retinal Circulation Time. 
Table 2. Changes of BCVA of the two groups ( $\mathrm{x} \pm \mathrm{s}$, letters).

\begin{tabular}{ccccc}
\hline & \multicolumn{2}{c}{ BCVA Results } & $\mathrm{t}$ & $\mathrm{p}$ \\
\cline { 2 - 5 } & Baseline & 6 months & 8.021 & 0.000 \\
\hline Treatment Group & $64 \pm 14$ & $74 \pm 10$ & 2.103 & 0.044 \\
\hline Control Group & $65 \pm 13$ & $67 \pm 13$ & \\
\hline
\end{tabular}

Data are mean $\pm \mathrm{SD}$; BCVA: Best-Corrected Visual Acuity.

Table 3. Changes of CRT of the two groups $(\mathrm{x} \pm \mathrm{s}, \mu \mathrm{m})$.

\begin{tabular}{ccccc}
\hline & \multicolumn{2}{c}{ OCT Results } & $\mathrm{t}$ & $\mathrm{p}$ \\
\cline { 2 - 5 } & Baseline & 6 months & & 0.007 \\
\hline Treatment Group & $406.3 \pm 23.3$ & $338.4 \pm 20.7$ & 2.571 & 0.07 \\
Control Group & $385.3 \pm 8.3$ & $342.7 \pm 19.2$ & 1.016 & 0.07 \\
\hline
\end{tabular}

Data are mean \pm SD; CRT: Central Retinal Thickness; OCT: Optical Coherence Tomography.

Table 4. Changes of RCT of the two groups $(x \pm s, s e c)$.

\begin{tabular}{ccccc}
\hline & \multicolumn{2}{c}{ RCT Results } & t & \\
\cline { 2 - 4 } & Baseline & 6 months & 43.83 & 0.000 \\
Treatment Group & $5.11 \pm 0.29$ & $3.04 \pm 0.14$ & 27.34 & 0.000 \\
Control Group & $5.15 \pm 0.21$ & $3.81 \pm 0.13$ & \\
\hline
\end{tabular}

Data are mean $\pm \mathrm{SD}$; RCT: Retinal Circulation Time.

\section{Discussion}

Retinal vascular occlusive disorders constitute one of the major causes of blindness and impaired vision. There is marked controversy on their pathogeneses, clinical features and particularly their management. Recently, advances in clinical research added anti-VEGF, corticosteroids and sustained-release implants to our armamentarium in the management of retinal vein occlusions [1]. Despite the existence of several therapeutic options, none is entirely satisfactory. Short-term effectiveness such as VA improvement may be significant, however, their long-term curative rates are still disappointing requiring expensive and repetitive treatments, for they only focus on the symptoms such as macular edema, not the disease. TCM has always been focusing on the diseases instead of the symptoms alone, so whether it may have some effectiveness on retinal vascular occlusive disorders is yet to be studied.

TCM is a unique system of theory, diagnosis and treatment tools, and is commonly used in Asian countries. Compared with western medicine, the TCM approach treats the function and dysfunction of living organisms in a more holistic way. However, the complexity of the chemical components and the actions in vivo often lead to great difficulties to elucidate the molecular mechanisms of TCM, which has been always the bottleneck of modern TCM study.

In this research, we studied TSA, which is an herbal monomer with a clear chemical structure, isolated from Danshen (Salvia miltiorrhiza). Danshen, a herbal medicine derived from the dried root of Salvia miltiorrhiza Bunge, is a hemorheologic agent that may have protective effect in patients with unstable angina [4] and has been used for cardiovascular disorders for hundreds of years in China and now is widely used in other countries as well. Danshen consists of a mixture of compounds, among which TSA represents the most biologically active ingredient [8]. Animal and cellular studies have shown various potential benefits of the agent, including neuroprotective effect in cerebral ischemia and reperfusion [9], antioxidant potential to prevent oxidation of low-density lipoproteins [10], reducing cellular damage by free radicals [11], protecting cardiomyocytes against oxidative stress-mediated apoptosis [12], and so on. Human studies also have demonstrated cardioprotective effects of TSA, including reduction of myocardial infarct size and decrease of myocardial consumption of oxygen [13]. TSA has drawn extensive attention because of its therapeutic efficacy in cardiovascular diseases, metabolic dis- 
eases as well as cancers [14]. As a multi-target drug, whether TSA may have protective effects on retinal vascular diseases has not been proved. Therefore, this study was designed to assess the safety and efficacy of TSA in treating RVO patients.

Here, we found that after 6 months SS injections, most patients in Treatment Group showed a good response with a significant reduction of CRT, RCT and an increase in VA. To date, initial VA is the most reliable prognostic factor of visual prognosis. Initial CRT can be useful for evaluating the severity of ME associated with $\mathrm{RVO}$, and seems to be important toward the presentation of visual function after treatment, while initial RCT can reflect the status of retinal circulation. During the follow-up period, no one was lost and all required data were collected in this trial.

This prospective study had a number of potential limitations. The sample size was small, the investigators and patients were not masked. Considering the strength of the evidence, more rigorously designed trials are required for assessing the effects of SS injection before it can be recommended routinely.

\section{Conclusion}

In summary, SS injection was an effective and safe modality for treating RVO patients based on the comparison of mean BCVA and OCT images and FFA results between the two groups. SS injection appeared to be an effective and safe treatment option for RVO.

\section{Acknowledgements}

This work was supported by a grant from Shanghai First People's Hospital. The author was grateful to Professor Wu from Shanghai First People's Hospital for final edict of this manuscript.

\section{References}

[1] Querques, G., Triolo, G., Casalino, G., García-Arumí, J., Badal, J., Zapata, M., Boixadera, A., Castillo, V.M. and Bandello, F. (2013) Retinal Venous Occlusions: Diagnosis and Choice of Treatments. Ophthalmic Research, 49, 215-222. http://dx.doi.org/10.1159/000346734

[2] Hahn, P. and Fekrat, S. (2012) Best Practices for Treatment of Retinal Vein Occlusion. Current Opinion in Ophthalmology, 23, 175-181. http://dx.doi.org/10.1097/ICU.0b013e3283524148

[3] Cernak, M. and Struharova, K. (2012) Current Therapy for Retinal Vein Occlusion. Bratisl Lek Listy, 113, $228-231$. http://dx.doi.org/10.4149/BLL_2012_052

[4] Zhou, L., Zuo, Z. and Chow, M.S. (2005) Danshen: An Overview of Its Chemistry, Pharmacology, Pharmacokinetics, and Clinical Use. The Journal of Clinical Pharmacology, 45, 1345-1359. http://dx.doi.org/10.1177/0091270005282630

[5] Chen, Y., Wu, X., Yu, S., Lin, X., Wu, J., Li, L., Zhao, J. and Zhao, Y. (2012 Neuroprotection of Tanshinone IIA against Cerebral Ischemia/Reperfusion Injury through Inhibition of Macrophage Migration Inhibitory Factor in Rats. PLoS ONE, 7, e40165. http://dx.doi.org/10.1371/journal.pone.0040165

[6] Fu, J., Huang, H., Liu, J., Pi, R., Chen, J. and Liu, P. (2007) Tanshinone IIA Protects Cardiac Myocytes against Oxidative Stress-Triggered Damage and Apoptosis. European Journal of Pharmacology, 568, 213-221. http://dx.doi.org/10.1016/j.ejphar.2007.04.031

[7] Park, O.K., Choi, J.H., Park, J.H., Kim, I.H., Yan, B.C., Ahn, J.H., Kwon, S.H., Lee, J.C., Kim, Y.S., Kim, M., Kang, I.J., Kim, J.D., Lee, Y.L. and Won, M.H. (2012) Comparison of Neuroprotective Effects of Five Major Lipophilic Diterpenoids from Danshen Extract against Experimentally Induced Transient Cerebral Ischemic Damage. Fitoterapia, 83, 1666-1674. http://dx.doi.org/10.1016/j.fitote.2012.09.020

[8] Shang, Q.H., Xu, H. and Huang, L. (2012) Tanshinone IIA: A Promising Natural Cardioprotective Agent. EvidenceBased Complementary and Alternative Medicine, 2012, Article ID: 716459. http://dx.doi.org/10.1155/2012/716459

[9] Chen, Y.L., Wu, X.M., Yu, S.S., Lin, X.M., Wu, J.X., Li, L., Zhao, J. and Zhao, Y. (2012) Neuroprotection of Tanshinone IIA against Cerebral Ischemia/Reperfusion Injury through Inhibition of Macrophage Migration Inhibitory Factor in Rats. PLoS ONE, 7, e40165. http://dx.doi.org/10.1371/journal.pone.0040165

[10] Li, X.X., Xu, X., Wang, J.N., Yu, H., Wang, X., Yang, H.J., Xu, H.Y., Tang, S.H., Li, Y., Yang, L., Huang, L.Q., Wang, Y.H. and Yang, S.L. (2012) A System-Level Investigation into the Mechanisms of Chinese Traditional Medicine: Compound Danshen Formula for Cardiovascular Disease Treatment. PLoS ONE, 7, e43918. http://dx.doi.org/10.1371/journal.pone.0043918

[11] Wang, A.-M., Sha, S.-H., Lesniak, W. and Schacht, J. (2003) Tanshinone (Salviae miltiorrhizae Extract) Preparations 
Attenuate Aminoglycoside-Induced Free Radical Formation in Vitro and Ototoxicity in Vivo. Antimicrobial Agents and Chemotherapy, 47, 1836-1841. http://dx.doi.org/10.1128/AAC.47.6.1836-1841.2003

[12] Zhang, M.-Q., Zheng, Y.-L., Chen, H., Tu, J.-F., Shen, Y., Guo, J.-P., Yang, X.-H., Yuan, S.-R., Chen, L.-Z., Chai, J.-J., Lu, J.-H. and Zhai, C.-L. (2013) Sodium Tanshinone IIA Sulfonate Protects Rat Myocardium against IschemiaReperfusion Injury via Activation of PI3K/Akt/FOXO3A/Bim Pathway. Acta Pharmacologia Sinica, 34, 1386-1396. http://dx.doi.org/10.1038/aps.2013.91

[13] Tan, X.Y., Li, J.P., Wang, X.Y., Chen, N., Cai, B.Z., Wang, G., Shan, H.L., Dong, D.L., Liu, Y.J., Li, X.D., Yang, F., Li, X., Zhang, P., Li, X.Q. Yang, B.F. and Lu, Y.J. (2011) Tanshinone IIA Protects against Cardiac Hypertrophy via Inhibiting Calcineurin/Nfatc3 Pathway. International Journal of Biological Sciences, 7, 383-389. http://dx.doi.org/10.7150/ijbs.7.383

[14] Xu, S. and Liu, P. (2013) Tanshinone II-A: New Perspectives for Old Remedies. Expert Opinion on Therapeutic Patents, 23, 149-153. http://dx.doi.org/10.1517/13543776.2013.743995 